\title{
Produção e caracterização de enzimas proteolíticas de Lentinus crinitus (L.) Fr. 1825 DPUA 1693 do bioma amazônico (Polyporaceae)
}

\section{Production and characterization of proteolytic enzymes of Lentinus crinitus (L.) Fr. 1825 DPUA 1693 from the Amazonian biome (Polyporaceae)}

\author{
Alessandra Alves da Silva Magalhães' (1) | Taciana de Amorim Silval (1) | \\ Maria Francisca Simas Teixeiral (1) | Raimundo Felipe Cruz Filho' (1) | \\ Suelen Dias da Silval (1) | Dib Mady Diniz Gomes' (1) | José Odair Pereiral (1) \\ 'Universidade Federal do Amazonas. Manaus, Amazonas, Brasil
}

\begin{abstract}
Resumo: Proteases são enzimas hidrolíticas capazes de degradar proteínas em pequenos peptídeos e aminoácidos, e podem ser isoladas de plantas, animais e microrganismos. A busca por proteases de origem microbiana vem aumentando no mercado mundial devido à sua estabilidade, à alta taxa de produção e ao menor custo que apresenta. O objetivo deste artigo foi investigar a produção de proteases por Lentinus crinitus (L.) Fr. 1825 DPUA 1693, utilizando-se a fermentação submersa. A cultura matriz foi preparada em meio BDA (ágar batata dextrose), adicionando-se extrato de levedura $0,5 \%(\mathrm{p} / \mathrm{v})$. A fermentação foi conduzida a $30^{\circ} \mathrm{C}, 150 \mathrm{rpm}$, por oito dias, utilizando-se meios contendo diferentes fontes de carbono e nitrogênio na síntese de proteases. Na determinação da atividade enzimática, foi adotada azocaseína $1 \%(\mathrm{p} / \mathrm{v})$ como substrato. Os resultados mostraram que a maior atividade das proteases de $L$. crinitus foi determinada no meio contendo glicose e gelatina. A atividade ótima dessas enzimas foi determinada em pH 5,0, a $50{ }^{\circ} \mathrm{C}$, e a estabilidade, em pH 5,0, à temperatura de $60^{\circ} \mathrm{C}$. As proteases foram classificadas como metalo, serino e cisteíno proteases. Este artigo sugere o uso potencial de protease de Lentinus crinitus para aplicação na indústria alimentícia, farmacêutica e cosmética.
\end{abstract}

Palavras-chave: Cogumelo. Fermentação submersa. Biocatalisador. Protease.

Abstract: Proteases are hydrolytic enzymes capable of degrading proteins in small peptides and amino acids and can be isolated from plants, animals, and microorganisms. The objective of this work was to investigate the production of proteases by Lentinus crinitus (L.) Fr. 1825 DPUA 1693, using fermentation in liquid medium. The matrix culture was prepared in BDA (potato dextrose agar) medium, added with $0.5 \%(\mathrm{w} / \mathrm{v})$ yeast extract. The fermentation was conducted at $30^{\circ} \mathrm{C}, 150 \mathrm{rpm}$ for eight days, using media containing different carbon and nitrogen sources in protease synthesis. In the determination of the enzymatic activity $1 \%$ azocasein $(w / v)$ was used as the substrate. The results showed that the increased activity of $L$. crinitus proteases was in the medium containing glucose and gelatin. The optimal activity of these enzymes was determined at pH 5.0 at $50^{\circ} \mathrm{C}$ and stability at pH 5.0 at $60^{\circ} \mathrm{C}$. Proteases were classified as metalo, serine, and cysteine proteases. This study suggests the potential use of Lentinus crinitus proteases for application in the food, pharmaceutical, and cosmetics industries.

Keywords: Mushroom. Submerged fermentation. Biocatalyst. Protease.

MAGALHÃES, A. A. S., T. A. SILVA, M. F. S. TEIXEIRA, R. F. CRUZ FILHO, S. D. SILVA, D. M. D. GOMES \&J. O. PEREIRA, 2019. Produção e caracterização de enzimas proteolíticas de Lentinus crinitus (L.) F. 1825 DPUA 1693 do bioma amazônico (Polyporaceae). Boletim do Museu Paraense Emílio Goeldi. Ciências Naturais 14(3): 453-461.

Autora para correspondência: Alessandra Alves da Silva Magalhães. Universidade Federal do Amazonas. Programa de Pós-Graduação da Rede de Biodiversidade da Amazônia Legal (BIONORTE). Av. General Rodrigo Otávio Jordão Ramos, 6200 - Coroado I. Manaus, AM, Brasil. CEP 69080-900 (alealvesmaga@yahoo.com.br).

Recebido em 01/03/2019

Aprovado em 21/06/2019

Responsabilidade editorial: Fernando da Silva Carvalho Filho
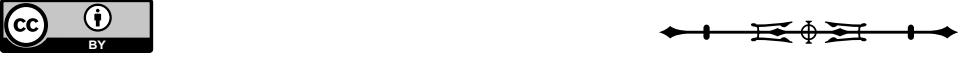


\section{INTRODUÇÃO}

Enzimas são catalisadores biológicos, em sua maioria de origem proteica, responsáveis por realizar catalisação de reações em organismos vivos (Sayali et al., 2013). Esses catalizadores são muito valorizados nas indústrias, pois são biodegradáveis, sendo derivados de fontes renováveis e apresentando reduzido impacto ambiental, devido à baixa quantidade de resíduos, características que despertam o interesse na busca por novas e mais eficientes fontes enzimáticas (Sheldon \& van Pelt, 2013).

As enzimas são biocatalisadores muito empregados em processos industriais e biotecnológicos, com grande relevância econômica no mercado mundial (Chapman et al., 2018). Recentemente, um relatório publicado pela BBC Research (2019) afirmou que a produção mundial de enzimas industriais alcançou um valor de US\$ 5,5 bilhões, em 2018, e a expectativa é de que este mercado atinja 7,0 bilhões de dólares até 2023.

Entre as enzimas, as proteases destacam-se por apresentar grande versatilidade de aplicações, sendo usadas em indústrias como as de produtos farmacêuticos (Kumar et al., 2015), alimentícios (Raveendran et al., 2018), de limpeza (Giri et al., 2011), no processo de amaciamento de couro (Singh \& Bajaj, 2017) e de biorremediação (Khan, 2013; Peralta et al., 2017). As proteases representam um dos maiores grupos de enzimas industriais, com perspectiva de aumento em torno de $7 \%$ até 2020 , e constituem $60 \%$ do mercado industrial global devido às suas propriedades biotecnológicas (Chandrasekaran et al., 2015; Bano et al., 2016; Singh et al., 2016).

Proteases são fisiologicamente importantes para a vida dos organismos, estando amplamente distribuídas na natureza, entretanto o seu processo de produção a partir de plantas e animais é mais oneroso e envolve políticas públicas e agrícolas que dificultam sua obtenção em larga escala, condição que promoveu interesse nestas enzimas de origem microbiana (Rao et al., 1998; Félix et al., 2004; Kumar et al., 2005).
A obtenção de proteases por microrganismos é possível graças à sua diversidade genética e bioquímica e por apresentarem fácil manipulação, o que viabiliza seu cultivo em grande escala (Rao et al., 1998; Kumar et al., 2005). Além disso, o processo de purificação e recuperação das proteases de origem microbiana é facilitado devido a esses microrganismos lançarem suas enzimas extracelulares diretamente no meio de fermentação (Savitha et al., 2011).

Entre os microrganismos, os fungos destacamse como fonte potencial de proteases, em razão de apresentarem grande capacidade de adaptação fisiológica, alta produtividade de biocompostos extracelulares, não dependência a condições ambientais e geográficas apropriadas, bem como possuírem gasto reduzido no uso de matérias-primas (Kumar et al., 2005; Zimmer et al., 2009; Peralta et al., 2017).

Os fungos do gênero Lentinus, pertencentes à família Lentinaceae (filo Basidiomycota), vêm se destacando devido à disporem de compostos bioativos, principalmente enzimas com aplicação industrial (Kirsch et al., 2013; Machado et al., 2016, 2017). Entretanto, a espécie Lentinus crinitus (L.) Fr., 1825, fungo sapróbio, lamelar e decompositor de madeira, ainda não foi estudada quanto ao seu potencial de produção de enzimas proteolíticas em condições de cultivo otimizadas.

Fatores como pH, temperatura e composição do meio são primordiais para obtenção de enzimas de interesse industrial (Abidi et al., 2011). A variação na relação de carbono e nitrogênio no meio de cultivo, a presença de íons de metal, a aeração, entre outros fatores físicos, são responsáveis pela otimização na produção de proteases (Joo \& Chang, 2005; Magalhães et al., 2017).

Considerando-se a importância da utilização de novas proteases obtidas a partir de cogumelos do bioma amazônico, o objetivo deste artigo foi avaliar o potencial de Lentinus crinitus na produção dessa enzima de interesse bioindustrial, conhecer a influência das fontes de carbono e nitrogênio e caracterizar as enzimas proteolíticas produzidas por este fungo.

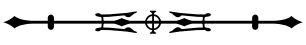




\section{MATERIAL E MÉTODOS}

\section{CULTIVO DO COGUMELO}

A linhagem Lentinus crinitus (L.) Fr. 1825 DPUA 1693 foi cedida pela Coleção de Culturas do Departamento de Parasitologia da Universidade Federal do Amazonas (DPUA). A cultura preservada em óleo mineral foi reativada em caldo glicosado (peptona de carne 1\%, extrato de levedura $0,3 \%$ e glicose $2 \%$ ) em cultivo estacionário, por 15 dias. Em seguida, foi realizado subcultivo em meio ágar batata dextrose (BDA), adicionado de extrato de levedura $0,5 \%(\mathrm{p} / \mathrm{v})$, utilizandose discos miceliais de $8 \mathrm{~mm}$ de diâmetro como inóculo em ponto central. $\bigcirc$ cultivo foi mantido a $25^{\circ} \mathrm{C}$, na ausência de luz, por oito dias (Kirsch et al., 2011).

\section{DETERMINAÇÃO DA INFLUÊNCIA DA FONTE DE CARBONO E DE NITROGÊNIO NA SÍNTESE DE PROTEASE EM CULTIVO SUBMERSO}

Para determinar a influência das fontes de carbono e de nitrogênio na produção de proteases, foram utilizadas várias formulações dos meios de cultivo conforme Kirsch et al. (2016), com modificações (Tabelas 1 e 2). Em todos os meios de cultivo, foi adicionado extrato de levedura 0,5\% (p/v) e o pH foi ajustado para 5,6.

Para produção de proteases, dez fragmentos miceliais de $8 \mathrm{~mm}$ de diâmetro foram inoculados em $100 \mathrm{~mL}$ dos meios de cultivo descritos na Tabela 1, distribuídos em frascos Erlenmeyer $(250 \mathrm{~mL})$. A fermentação submersa foi realizada a $30{ }^{\circ} \mathrm{C}, 150 \mathrm{rpm}$. Após oito dias, a biomassa foi separada por filtração sob vácuo em papel de filtro Whatman n. 1; em seguida, foram realizadas filtrações em membrana de éster de celulose, de 0,45 $\mu \mathrm{m}$ e 0,22 $\mu \mathrm{m}$. Na determinação da atividade proteolítica, $150 \mu \mathrm{L}$ do extrato bruto foi adicionado em $250 \mu \mathrm{L}$ de azocaseína 1\% (p/v), em solução-tampão Tris- $\mathrm{HCl} \mathrm{0,1} \mathrm{M,} \mathrm{pH} \mathrm{7,2.} \mathrm{A} \mathrm{mistura} \mathrm{reacional} \mathrm{foi} \mathrm{incubada}$ a $25^{\circ} \mathrm{C}$, em câmara escura. Após uma hora, a reação foi interrompida pela adição de 1,2 mL de ácido tricloroacético
Tabela 1. Meios de cultivo utilizados para a determinação da melhor fonte de carbono e nitrogênio para a produção de proteases. Legendas: GGE = glicose, gelatina e extrato de levedura; $\mathrm{AGE}=$ amido, gelatina e extrato de levedura; $\mathrm{SGE}=$ sacarose, gelatina e extrato de levedura; GPE = glicose, peptona e extrato de levedura; APE = amido, peptona e extrato de levedura; SPE = sacarose, peptona e extrato de levedura; GSE = glicose, sulfato de amônio e extrato de levedura; ASE = amido, sulfato de amônio e extrato de levedura; SSE = sacarose, sulfato de amônio e extrato de levedura.

\begin{tabular}{c|c|c}
\hline Meios & $\begin{array}{c}\text { Fonte de carbono } \\
2 \%(\mathrm{p} / \mathrm{v})\end{array}$ & $\begin{array}{c}\text { Fonte de nitrogênio } \\
0,5 \%(\mathrm{p} / \mathrm{v})\end{array}$ \\
\hline GGE & Glicose & Gelatina \\
\hline AGE & Amido & Gelatina \\
\hline SGE & Sacarose & Gelatina \\
\hline GPE & Glicose & Peptona \\
\hline APE & Amido & Peptona \\
\hline SPE & Sacarose & Peptona \\
\hline GSE & Glicose & Sulfato de amônio \\
\hline ASE & Amido & Sulfato de amônio \\
\hline SSE & Sacarose & Sulfato de amônio \\
\hline
\end{tabular}

10\% (p/v). Do sobrenadante, foram transferidos 800 $\mu \mathrm{L}$ para 1,4 $\mathrm{mL}$ de hidróxido de sódio $1 \mathrm{M}$. A atividade enzimática foi determinada de acordo com a metodologia recomendada por Porto et al. (1996), que definem uma unidade de atividade de protease como a quantidade de enzima necessária para produzir variação de absorbância igual a 0,001 em 60 minutos. Todos os experimentos foram realizados em triplicata.

\section{EFEITOS DO PH E DA TEMPERATURA SOBRE A} ATIVIDADE PROTEOLÍTICA

$\mathrm{O}$ pH ótimo foi determinado a $25^{\circ} \mathrm{C}$ em diferentes valores de $\mathrm{pH}$, utilizando-se as seguintes soluções-tampão a 0,1 M: acetato de sódio (para pH 5), fosfato (para 6, 7 e 8) e glicina$\mathrm{NaOH}(9$ e 10). A temperatura ótima foi determinada pela incubação do extrato bruto em diferentes temperaturas, que variaram de 30 a $80{ }^{\circ} \mathrm{C}$. A determinação da atividade proteolítica foi realizada conforme metodologia descrita anteriormente (Merheb-Dini et al., 2010).

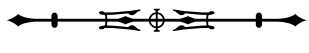




\section{EFEITOS DO PH E DA TEMPERATURA SOBRE} A ESTABILIDADE PROTEOLÍTICA

Para conferir estabilidade ao $\mathrm{pH}$, o extrato bruto foi diluído (1:1) nas seguintes soluções-tampão a 0,1 M: acetato de sódio ( $\mathrm{pH}$ 5), fosfato $(\mathrm{pH} \mathrm{6,7} \mathrm{e} \mathrm{8)} \mathrm{e}$ glicina- $\mathrm{NaOH}\left(\mathrm{pH} 9\right.$ e 10), mantidas a $25^{\circ} \mathrm{C}$, por 24 horas, seguindo-se da determinação da atividade proteolítica nas condições ótimas de $\mathrm{pH}$ e temperatura encontradas. Para a realização do teste de estabilidade à temperatura, incubou-se cada extrato enzimático durante $1 \mathrm{~h}$ em diferentes temperaturas $(30,40,50,60$, $70,8{ }^{\circ} \mathrm{C}$ ), seguindo-se da determinação da atividade proteolítica em condições ótimas de $\mathrm{pH}$ e temperatura. A determinação da atividade proteolítica foi realizada conforme metodologia descrita anteriormente, e os resultados foram expressos em atividade relativa em todos os experimentos (Merheb-Dini et al., 2010).

\section{EFEITO DE ÍONS METÁLICOS E INIBIDORES NA ATIVIDADE PROTEOLÍTICA}

Para investigar as possíveis aplicações das proteases de Lentinus crinitus, foram analisados os efeitos dos seguintes íons metálicos sobre a atividade proteolítica: $\mathrm{CaCl}_{2}, \mathrm{CuSO}_{4}, \mathrm{FeSO}_{4}, \mathrm{MgSO}_{4}, \mathrm{MnSO}_{4}, \mathrm{ZnSO}_{4}, \mathrm{NaCl}$ e $\mathrm{KCl}$. Também foram analisados os efeitos dos seguintes inibidores: fluoreto de fenilmetanosulfonila (PMSF), ácido etilenodiaminotetracético (EDTA), ácido iodoacético e pepstatin A. A atividade proteolítica das enzimas do cogumelo foi determinada após incubação do extrato nas condições ótimas de temperatura e pH, com uma solução de cada íon/inibidores na concentração de 10 mM (com exceção da pepstatin $A[1 \mathrm{mM}])$. A atividade enzimática residual foi determinada e comparada com o controle que corresponde a 100\% de atividade. Todas as amostras foram preparadas em triplicata (Alecrim et al., 2015; Martim et al., 2017).

\section{ANÁLISE ESTATÍSTICA}

Em todos os experimentos, os dados foram submetidos à análise de variância, e as médias foram comparadas pelo teste de Tukey $(P<0,05)$, por meio do programa Minitab, versão 18.1 (Minitab, 2017).

\section{RESULTADOS E DISCUSSÃO}

\section{PRODUÇÃO DE PROTEASES POR}

\section{FERMENTAÇÃO SUBMERSA EM DIFERENTES} FONTES DE CARBONO E DE NITROGÊNIO

A Tabela 2 demonstra a influência das fontes de carbono e de nitrogênio. Em todos os meios testados, houve produção de proteases, porém a maior atividade (190 U/mL $\pm 1,33$ ) foi observada no meio GGE [glicose $2 \%(\mathrm{p} / \mathrm{v})+$ gelatina $0,5 \%(\mathrm{p} / \mathrm{v})+$ extrato de levedura $0,5 \%(\mathrm{p} / \mathrm{v})]$, enquanto a menor atividade $(77,33 \mathrm{U} / \mathrm{mL} \pm$ 2,31), no meio ASE [amido $2 \%(\mathrm{p} / \mathrm{v})+$ sulfato de amônio $0,5 \%(\mathrm{p} / \mathrm{v})+$ extrato de levedura $0,5 \%(\mathrm{p} / \mathrm{v})]$.

Martim et al. (2017) estudaram a atividade proteolítica de cogumelos amazônicos - Pleurotus albidus (Berk.) Pegler 1983 DPUA 1692, Lentinus crinitus (L.) Fr. 1825 DPUA 1693 e Ganoderma lucidum (Curtis) P. Karst 1881 DPUA 1694 - e obtiveram a maior atividade enzimática com o

Tabela 2. Produção de proteases de Lentinus crinitus em diferentes meios de cultura. Letras iguais na coluna não diferem estatisticamente pelo teste de Tukey $(P<0,05)$. Legendas: $G G E=$ glicose, gelatina e extrato de levedura; AGE = amido, gelatina e extrato de levedura; SGE = sacarose, gelatina e extrato de levedura; GPE = glicose, peptona e extrato de levedura; APE = amido, peptona e extrato de levedura; SPE = sacarose, peptona e extrato de levedura; GSE = glicose, sulfato de amônio e extrato de levedura; ASE = amido, sulfato de amônio e extrato de levedura; SSE = sacarose, sulfato de amônio e extrato de levedura.

\begin{tabular}{c|c}
\hline Meios & Atividade proteolítica $(\mathrm{U} / \mathrm{mL})$ \\
\hline GGE & $190,00^{\mathrm{a}} \pm 1,33$ \\
\hline AGE & $156,67^{\mathrm{b}} \pm 2,00$ \\
\hline SGE & $153,78^{\mathrm{b}} \pm 1,02$ \\
\hline GPE & $147,11^{\mathrm{c}} \pm 1,02$ \\
\hline APE & $127,78^{\mathrm{d}} \pm 1,68$ \\
\hline SPE & $127,33^{\mathrm{d}} \pm 1,33$ \\
\hline GSE & $88,00^{\mathrm{e}} \pm 1,15$ \\
\hline ASE & $88,00^{\mathrm{e}} \pm 2,00$ \\
\hline SSE & $77,33^{\mathrm{f}} \pm 2,31$ \\
\hline
\end{tabular}

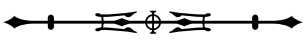


fungo Pleurotus albidus $(34,00 \mathrm{U} / \mathrm{mL} \pm 0,0)$. Eles obtiveram atividade proteolítica de Lentinus crinitus igual a 27,02 $U / \mathrm{mL} \pm 0,02$, valor bem inferior ao obtido no presente artigo (190 U/mL $\pm 1,33)$, demonstrando a importância do estudo do efeito de diferentes fontes de carbono e de nitrogênio na produção de enzimas de valor industrial.

Neste artigo, observou-se que a gelatina foi a melhor fonte de nitrogênio para produção de protease em Lentinus crinitus - meio GGE (190,00 \pm 1,33). A produção de proteases depende da disponibilidade de carbono e de fontes de nitrogênio do meio, sendo que ambos os parâmetros exercem efeitos reguladores sobre a síntese da enzima (Haddar et al., 2010). A definição da fonte de nitrogênio de um bioprocesso é um dos fatores mais importantes a ser investigado na síntese proteica. Kirsch et al. (2011) também relataram, por meio do cogumelo Lentinus citrinus, que a gelatina se destacou como a melhor fonte de nitrogênio para a produção das proteases.

Entretanto, os ensaios contendo sulfato de amônio como fonte de nitrogênio (SSE e ASE) foram os que produziram menor quantidade de protease. Fang \& Zhong (2002), comparando o efeito de fontes inorgânicas de nitrogênio com fontes orgânicas sobre a produção de biomoléculas do cogumelo Ganoderma lucidum, verificaram maior crescimento micelial nos meios contendo nitrogênio orgânico, sugerindo que certos aminoácidos essenciais não seriam sintetizados por células de fungos superiores a partir de fontes de nitrogênio inorgânico, como o sulfato de amônio.

\section{EFEITO DO PH E DA TEMPERATURA NA ATIVIDADE PROTEOLÍTICA DE LENTINUS CRINITUS}

$O$ efeito do $\mathrm{pH}$ na atividade enzimática foi avaliado na faixa de 5 a 10 (Figura 1). Em pH ácido, as proteases de Lentinus crinitus apresentaram o melhor desempenho, com 75\% de atividade no $\mathrm{pH}$ 6,0 e 100\% de atividade enzimática no pH 5,0. Nos pH com variação de neutro a alcalino, observou-se redução da atividade proteolítica apresentando percentagem de $36 \%$ no pH 10. Esses dados corroboram os resultados encontrados por Martim et al. (2017), que avaliaram o extrato bruto do cogumelo amazônico Pleurotus albidus, verificando que a maior atividade proteolítica ocorreu no pH 5,0. Kirsch et al. (2013), analisando o cultivo submerso do cogumelo Lentinus citrinus ocorrente na Amazônia, verificaram que o $\mathrm{pH}$ 7,0 foi o mais promissor para atividade proteolítica.

Estudos enfatizam a importância do controle de fatores externos, como $\mathrm{pH}$ e temperatura, para assegurar a manutenção das condições ótimas de cultivo do microrganismo e da atividade biológica da enzima, uma vez que essas enzimas podem vir a sofrer desnaturação quando submetidas a determinadas condições ambientais (Bon et al., 2008).

O efeito da temperatura na atividade enzimática foi avaliado entre 30 a $80^{\circ} \mathrm{C}$, com intervalo de $10^{\circ} \mathrm{C}$ (Figura 2). Nestas condições, as enzimas de L. crinitus demonstraram atividade ótima a $50^{\circ} \mathrm{C}$, seguida de decréscimo nas temperaturas mais elevadas. Em temperaturas elevadas, as enzimas sofrem desnaturação proteica, pois as ligações intramoleculares são afetadas (Ali et al., 2014). Estes resultados estão de acordo com Zhang et al. (2010) e Guan et al. (2011), em que a temperatura ótima das proteases de Hypsizigus marmoreus (Peck) H.E. Bigelow 1976 e Pholiota nameko (T. Itô) S. Ito \& S. Imai 1933, respectivamente, foi determinada a $50{ }^{\circ} \mathrm{C}$.

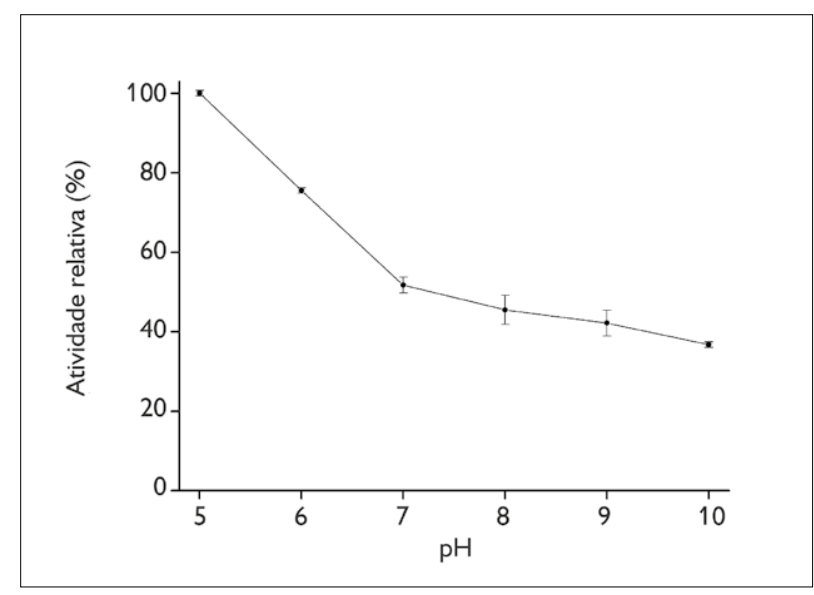

Figura 1. Efeito do pH na atividade proteolítica de Lentinus crinitus.

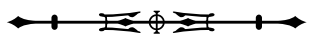




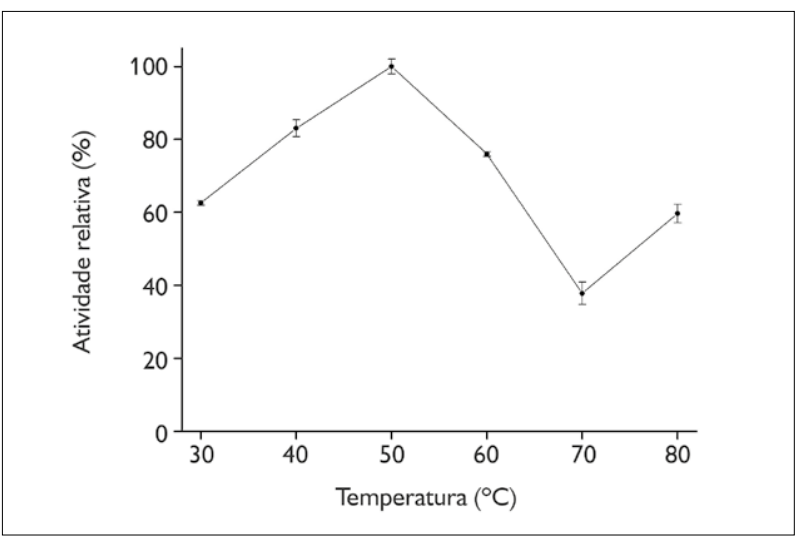

Figura 2. Efeito da temperatura na atividade proteolítica de Lentinus crinitus.

\section{EFEITO DO PH E DA TEMPERATURA NA ATIVIDADE E NA ESTABILIDADE PROTEOLÍTICA DE LENTINUS CRINITUS}

As enzimas proteolíticas de Lentinus crinitus apresentaram boa estabilidade em pH ácido (pH 5,0 e 6,0), retendo, em média, 90,59\% de atividade enzimática, enquanto que a média de retenção da atividade proteolítica foi de 32,25\% em pH alcalino ( $\mathrm{pH}$ 8,0, 9,0 e 10,0), conforme os dados da Figura 3. Martim et al. (2017), ao analisarem as proteases do extrato bruto do cogumelo amazônico Pleurotus albidus, verificaram elevada estabilidade da enzima entre $\mathrm{pH}$ de 5 a 8, com redução da atividade em pH 9 e 10. Entretanto, Kirsch et al. (2013), ao investigarem a estabilidade do extrato bruto do cogumelo Lentinus citrinus de ocorrência na Amazônia, observaram estabilidade em valores de $\mathrm{pH}$ de 5 a 7 , e queda na estabilidade no $\mathrm{pH} 10$.

A estabilidade da atividade proteolítica de Lentinus crinitus em diferentes temperaturas $\left(30\right.$ a $\left.80^{\circ} \mathrm{C}\right)$ pode ser observada na Figura 4. A estabilidade térmica destas enzimas foi mantida entre 50 a $60^{\circ} \mathrm{C}$, exibindo 93,35 a $100 \%$ de atividade relativa, respectivamente. $\mathrm{Em} 80^{\circ} \mathrm{C}$ foi observado decréscimo acentuado da atividade da enzima, com retenção de apenas 29,49\% de atividade relativa.

Machado et al. (2017), avaliando a estabilidade a diferentes temperaturas do extrato bruto de Pleurotus

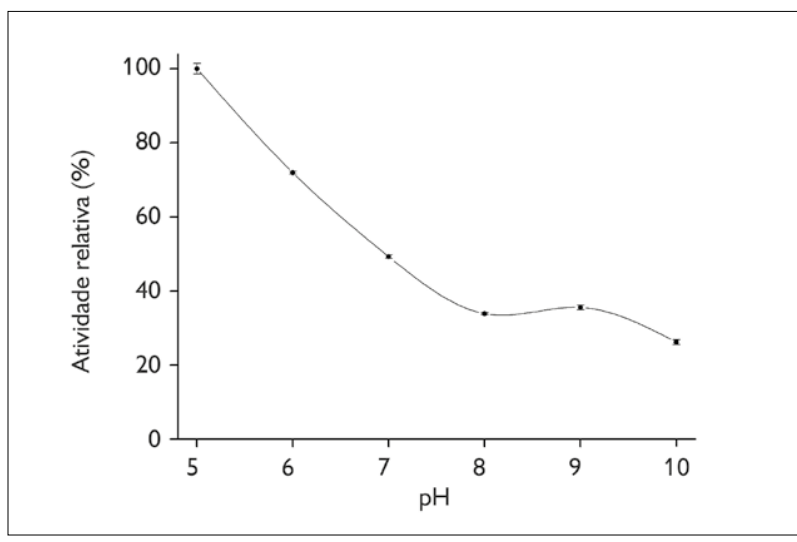

Figura 3. Efeito do $\mathrm{pH}$ na estabilidade da atividade proteolítica de Lentinus crinitus.

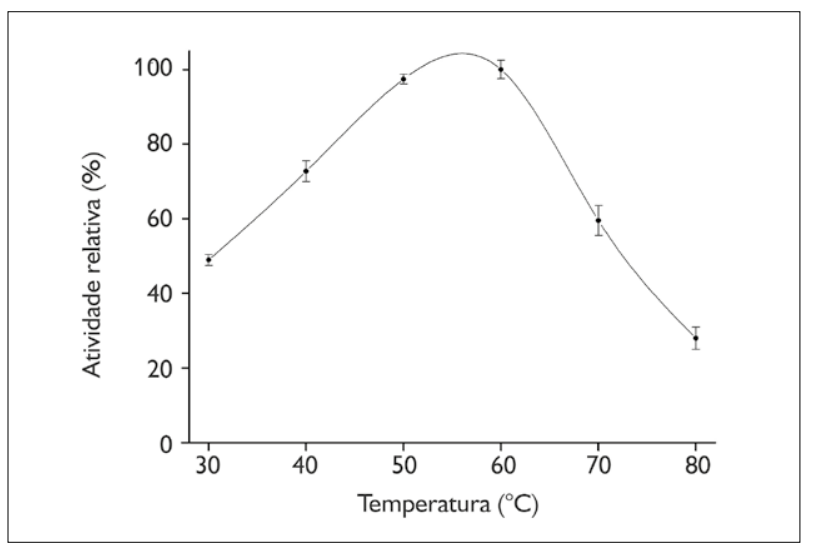

Figura 4. Efeito da temperatura na estabilidade da atividade proteolítica de Lentinus crinitus.

ostreatoroseus Singer 1961, verificaram que a estabilidade foi mantida entre 40 e $50^{\circ} \mathrm{C}$, e que a inativação da enzima ocorreu a $70^{\circ} \mathrm{C}$. Estudos mostram que a estabilidade ao $\mathrm{pH}$ e à temperatura são parâmetros importantes para aplicação industrial das enzimas, pois determinam a viabilidade econômica do processo (Gupta \& Ayyachamy, 2012; Moretti et al., 2012).

\section{EFEITO DE ÍONS METÁLICOS E INIBIDORES NA ATIVIDADE PROTEOLÍTICA DE LENTINUS CRINITUS}

A atividade proteolítica relativa das enzimas de Lentinus crinitus foi reduzida significativamente na presença de EDTA 
(60,51\%), ácido iodo acético $(62,61 \%)$ e fluoreto de fenilmetanosulfonila (PMSF) (64,86\%), conforme dados da Tabela 3. Esse resultado sugere que as enzimas testadas são sensíveis aos três inibidores supracitados, indicando que o extrato bruto do fungo contém metalo proteases, cisteíno proteases e serino proteases, respectivamente. Entretanto, não houve retenção significativa da atividade enzimática na presença de pepstatin $A$, indicando a ausência de resíduos de ácido aspártico no sítio ativo da enzima.

Resultados semelhantes foram encontrados por Machado et al. (2017), os quais relatam a presença de cisteíno proteases, serino protease e metalo proteases em extratos de Pleurotus ostreatoroseus. Martim et al. (2017), analisando o efeito de inibidores na atividade proteolítica do extrato bruto do cogumelo amazônico Pleurotus albidus, também verificaram a presença de serino e cisteíno proteases no extrato bruto do cogumelo.

A maioria dos íons testados interagiu com o sítio ativo das enzimas reduzindo sua atividade catalítica, conforme a Tabela 3 demonstra. A menor atividade relativa da enzima foi verificada na presença de $\mathrm{Fe}^{2+}(31,08 \%)$, e os íons $\mathrm{K}^{+}$,

Tabela 3. Efeito de íons metálicos na atividade proteolítica de Lentinus crinitus. Letras iguais na coluna não diferem estatisticamente pelo teste de Tukey $(P<0,05)$.

\begin{tabular}{c|c}
\hline Íon metálico ou inibidor & Atividade relativa (\%) \\
\hline $\mathrm{Mn}^{+2}$ & $129,43^{\mathrm{a}} \pm 3,15$ \\
\hline $\mathrm{Cu}^{+2}$ & $78,60^{\mathrm{b}} \pm 3,00$ \\
\hline $\mathrm{Mg}^{+2}$ & $42,79^{\mathrm{c}} \pm 2,40$ \\
\hline $\mathrm{Zn}^{+2}$ & $38,29^{\mathrm{d}} \pm 3,33$ \\
\hline $\mathrm{Ca}^{+2}$ & $36,04^{\mathrm{de}} \pm 1,33$ \\
\hline $\mathrm{Na}^{+}$ & $33,93^{\mathrm{ef}} \pm 1,54$ \\
\hline $\mathrm{K}^{+}$ & $32,28^{\mathrm{ef}} \pm 1,02$ \\
\hline Fe & $31,08^{\mathrm{f}} \pm 0,67$ \\
\hline Pepstatin A & $93,54^{\mathrm{a}} \pm 2,78$ \\
\hline EDTA & $39,49^{\mathrm{b}} \pm 2,04$ \\
\hline Ácido iodoacético & $36,79^{\mathrm{bc}} \pm 1,68$ \\
\hline $\begin{array}{c}\text { Fluoreto de } \\
\text { fenilmetanosulfonila }\end{array}$ & $34,98^{\mathrm{c}} \pm 2,34$ \\
\hline
\end{tabular}

$\mathrm{Ca}^{2+}, \mathrm{Na}^{+}, \mathrm{Mg}^{2+}$ e $\mathrm{Zn}^{2+}$ reduziram significativamente a atividade da enzima para valores inferiores a $43 \%$ de atividade relativa. Em condições similares, $\mathrm{Cu}^{2+}$ exerceu pouca influência na atividade enzimática, retendo 78,60\%. Entretanto, na presença de $\mathrm{Mn}^{2+}$, a atividade proteolítica de L. crinitus foi potencializada (129,43\%).

Harer et al. (2018) afirmam que alguns íons metálicos como $\mathrm{Ca}^{+2}, \mathrm{Mg}^{+2}$ e $\mathrm{Mn}^{+2}$ aumentam e estabilizam a atividade enzimática. Os íons podem estar envolvidos nos processos catalíticos, participando das reações de redox ou de transferência de elétrons (Nazmi et al., 2006). Esses íons metálicos protegem a enzima contra a desnaturação térmica e desempenham papel vital na manutenção da conformação ativa da enzima a temperaturas mais elevadas (Gupta et al., 2002).

\section{CONCLUSÃO}

Os resultados desta pesquisa indicam que Lentinus crinitus produz maior quantidade de protease quando cultivado em meios contendo fontes orgânicas de nitrogênio, e que os melhores nutrientes para induzir a produção de enzimas proteolíticas por fermentação submersa, nas condições testadas, foram glicose e gelatina. As proteases de L. crinitus são predominantemente ácidas, termoestáveis, dos tipos metalo, serino e cisteíno proteases, com atividade ótima a $50^{\circ} \mathrm{C}$, em pH 5,0, e maior estabilidade a $60^{\circ} \mathrm{C}$. Este estudo sugere o uso potencial de proteases de L. crinitus para aplicação em indústrias de alimentos, bebidas e produtos farmacêuticos, representando uma alternativa viável, de fonte segura e sustentável, para o polo industrial da região amazônica.

\section{AGRADECIMENTOS}

À Coordenação de Aperfeiçoamento de Pessoal de Nível Superior (CAPES), pelo financiamento da pesquisa; à Universidade Federal do Amazonas (UFAM), ao Programa de Pós-Graduação da Rede de Biodiversidade da Amazônia Legal (BIONORTE) e ao professor Dr. Adolfo J. Mota (UFAM), pelo apoio técnico, científico e financeiro.

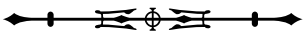




\section{REFERÊNCIAS}

ABIDI, F., J. M. CHOBERT, T. HAERTLÉ \& M. N. MARZOUKI, 2011. Purification and biochemical characterization of stable alkaline protease Prot-2 from Botrytis cinerea. Process Biochemistry 46(12): 2301-2310. DOI: https://doi.org/10.1016/j.procbio.2011.09.010.

ALECRIM, M. M., R. A. PALHETA, M. F. S. TEIXEIRA \& I. M. D. A. OLIVEIRA, 2015. Milk- clotting enzymes produced by Aspergillus flavo furcatis strains on Amazonic fruit waste. International Journal of Food Science \& Technology 50(1): 151-157. DOI: https://doi. org/10.1111/ijfs. 12677 .

ALI, S. M., T. C. LING, S. MUNIANDY, Y. S. TAN, J. RAMAN \& V. SABARATNAM, 2014. Recovery and partial purification of fibrinolytic enzymes of Auricularia polytricha (Mont.) Sacc by an aqueous twophase system. Separation and Purification Technology 122(1): 359-366. DOI: https://doi.org/10.1016/j.seppur.2013.11.016.

BANO, S., M. U. DAHOT \& S. H. A. NAQVI, 2016. Optimization of culture conditions for the production of protease by Pleurotus eryngii. Pakistan Journal of Biotechnology 13(3): 193-198.

BBC RESEARCH, 2019. Global markets for enzymes in industrial applications. Disponível em: http://bbcresearch.com/. Acesso em: 14 fevereiro 2019.

BON, E. P. S., N. PEREIRA JR., L. M. F. GOTTSCHALK, P. SÁPEREIRA, J. C. ROSEIRO \& M. A. FERRARA, 2008. Bioprocessos para produção de enzimas. In: E. P. S. BOM, M. A. FERRARA \& M. L. CORVO (Ed.): Enzimas em biotecnologia: produção, aplicação e mercado: 95-122. Interciências Ltda., Rio de Janeiro.

CHANDRASEKARAN, S., S. S. P. KUMARESAN \& M. MANAVALAN, 2015. Production and optimization of protease by filamentous fungus isolated from paddy soil in Thiruvarur District Tamilnadu. Journal of Applied Biology \& Biotechnology 3(6): 66-69. DOI: https://doi. org/10.7324/JABB.2015.3610.

CHAPMAN, J., A. E. ISMAIL \& C. Z. DINU, 2018. Industrial applications of enzymes: recent advances, techniques, and outlooks. Catalysts 8(6): 238. DOI: https://doi.org/10.3390/catal8060238.

FANG, Q. H. \& J. J. ZHONG, 2002. Submerged fermentation of higher fungus Ganoderma lucidum for production of valuable bioactive metabolites-ganoderic acid and polysaccharide. Biochemical Engineering Journal 10(1): 61-65. DOI: https://doi.org/10.1016/ S1369-703X(01)00158-9.

FÉLIX, C. R., E. F. NORONHA \& L. MARCO, 2004. Proteases: características e aplicações industriais. In: L. SUMMA (Ed.): Enzimas como agentes biotecnológicos. 327-347. Editora Legis Summa, São Paulo.

GIRI, S. S., S. SUKUMARAN, S. S. SEN, M. OVIYA, N. BANU \& P. K. JENA, 2011. Purification and partial characterization of a detergent and oxidizing agent stable alkaline protease from a newly isolated Bacillus subtilis VSG-4 of tropical soil. Journal of Microbiology 49(3): 455-461. DOI: https://doi.org/10.1007/s12275-011-0427-4.
GUAN, G. P., G. Q. ZHANG, Y. Y. WU, H. X. WANG \& T. B. NG, 2011. Purification and characterization of a novel serine protease from the mushroom Pholiota nameko. Journal of Bioscience and Bioengineering 111(6): 641-645. DOI: https://doi.org/10.1016/j. jbiosc.2011.02.009.

GUPTA, R., Q. BEG, S. KHAN \& B. CHAUHAN, 2002. An overview on fermentation, downstream processing and properties of microbial alkaline proteases. Applied Microbiology and Biotechnology 60(4): 381-395. DOI: https://doi.org/10.1007/ s00253-002-1142-1.

GUPTA, K. \& M. AYYACHAMY, 2012. Biotechnology of fungal genes: 1-400. Taylor \& Francis Group, CRC Press, Boca Raton.

HADDAR, A., N. FAKHFAKH-ZOUARI, N. HMIDET, F. FRIKHA, M. NASRI \& A. S. KAMOUN, 2010. Low-cost fermentation medium for alkaline protease production by Bacillus mojavensis A21 using hulled grain of wheat and sardinella peptone. Journal of Bioscience and Bioengineering 110(3): 288-294. DOI: http:// dx.doi.org/10.1016/j.jbiosc.2010.03.015.

HARER, S. L., M. S. BHATIA \& N. M. BHATIA, 2018. Isolation, purification and partial characterization of thermostable serine alkaline protease from a newly isolated Bacillus thuringinsis-SH-II1A. African Journal of Biotechnology 17(7): 178-188. DOI: http:// dx.doi.org/10.5897/AJB2015.14831.

JOO, H. S. \& C. S. CHANG, 2005. Production of protease from a new alkalophilic Bacillus sp. I-312 grown on soybean meal: optimization and some properties. Process Biochemistry 40(3-4): 1263-1270. DOI: https://doi.org/10.1016/j.procbio.2004.05.010.

KHAN, F., 2013. New microbial proteases in leather and detergent industries. Innovation Research of Chemistry 1(1): 1-6.

KIRSCH, L. S., A. C. PINTO, T. S. PORTO, A. L. PORTO \& M. F. S. TEIXEIRA, 2011. The influence of different submerged cultivation conditions on mycelial biomass and protease production by Lentinus citrinus Walleyn et Rammeloo DPUA 1535 (Agaricomycetidae). International Journal of Medicinal Mushrooms 13(2): 185-192. DOI: http://dx.doi.org/10.1615/IntJMedMushr.v13.i2.

KIRSCH, L. S., V. C. S EBINUMA \& M. F. S. TEIXEIRA, 2013. Mycelial biomass and biochemical properties of proteases produced by Lentinus citrinus DPUA 1535 (Higher Basidiomycetes) in submerged cultivation. International Journal of Medicinal 15(5): 505-515. DOI: https://doi.org/10.1615/IntJMedMushr.v15.i5.80.

KIRSCH, L. S., A. J. P. MACEDO \& M. F. S. TEIXEIRA, 2016. Production of mycelial biomass by the Amazonian edible mushroom Pleurotus albidus. Brazilian Journal of Microbiology 47(3): 658664. DOI: http://dx.doi.org/10.1016/j.bjm.2016.04.007.

KUMAR, S., N. S. SHARMA, M. R. SAHARAN \& R. SINGH, 2005. Extracellular acid protease from Rhizopus oryzae: purification and characterization. Process Biochemistry 40(5): 1701-1705. DOI: https://doi.org/10.1016/j.procbio.2004.06.047.

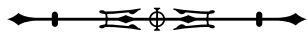


KUMAR, R. S., R. RAJESH, S. GOKULAKRISHNAN \& I. SUBRAMANIAN, 2015. Screening and characterization of fibrinolytic protease producing Bacillus circulans from mangrove sediments Pitchavaram, South East Coast of India. International Letters of Natural Sciences 28: 10-16. DOI: https://doi.org/10.18052/www. scipress.com/ILNS.28.10.

MACHADO, A. R. G., M. F. S. TEIXEIRA, L. S. KIRSCH, M. C. L. CAMPELO \& I. M. A. OLIVEIRA, 2016. Nutritional value and proteases of Lentinus citrinus produced by solid state fermentation of lignocellulosic waste from tropical region. Saudi Journal of Biological Sciences 23(5): 621-627. DOI: https://doi.org/10.1016/j. sjbs.2015.07.002.

MACHADO, A. R. G., S. R. MARTIM, M. M. ALECRIM \& M. F. S. TEIXEIRA, 2017. Production and characterization of proteases from edible mushrooms cultivated on amazonic tubers. African Journal of Biotechnology 16(46): 2160-2166. DOI: https://doi. org/10.5897/AJB2017.16154.

MAGALHÃES, A. A. S., T. B. CARVALHO, A. Q. L. SOUZA \& J. O. PEREIRA, 2017. Efeito da temperatura e do $\mathrm{pH}$ no crescimento micelial do fungo amazônico Lentinus crinitus (L.) Fr. In: L. A. OLIVEIRA, J. L. S. BENTES, M. A. JESUS, L. C. ROCHA, O. C. FERNANDES, S. L. ANDRADE \& A. Q. L. SOUZA (Org.): Diversidade microbiana da Amazônia: 2: 200207. INPA, Manaus.

MARTIM, S. R., L. S. C. SILVA, M. M. ALECRIM, B. C. SOUZA, I. M. A. OLIVEIRA \& M. F. S. TEIXEIRA, 2017. Proteases ácidas de cogumelo comestível da Amazônia para aplicabilidade industrial. Boletim do Museu Paraense Emílio Goeldi. Ciências Naturais 12(3): 353-362.

MERHEB-DINI, C., E. GOMES, M. BOSCOLO \& R. SILVA, 2010. Production and characterization of a milk-clotting protease in the crude enzymatic extract from the newly isolated Thermomucor indicae-seudaticae N31: (milk-clotting protease from the newly isolated Thermomucor indicae-seudaticae N31). Food Chemistry 120(1): 87-93. DOI: https://doi.org/10.1016/j. foodchem.2009.09.075.

MINITAB, 2017. Minitab statistical software. Version 18.1. LEAD Technologies, Inc., [S. I].

MORETTI, M., D. A. BOCCHINI-MARTINS, R. D. SILVA, A. RODRIGUES, L. D. SETTE \& E. GOMES, 2012. Selection of thermophilic and thermotolerant fungi for the production of cellulases and xylanases under solid-state fermentation. Brazilian Journal of Microbiology 43(3): 1062-1071. DOI: http://dx.doi. org/10.1590/S1517-83822012000300032.

NAZMI, A. R., T. REINISCH \& H. J. HINZ, 2006. Ca-binding to Bacillus licheniformis $\alpha$-amylase (BLA). Archives of Biochemistry and Biophysics 453(1): 18-25. DOI: https://doi.org/10.1016/j. abb.2006.04.004.
PERALTA, R. M., B. P. SILVA, R. C. G. CÔRREA, C. G. KATO, F. A. V. SEIXAS \& A. BRACHT, 2017. Enzymes from Basidiomycetes peculiar and efficient tools for biotechnology. In: G. BRAHMACHARI (Ed.): Biotechnology of microbial enzymes: 119-149. Academic Press, New York. DOI: http://dx.doi.org/10.1016/B978-0-12803725-6.00005-4.

PORTO, A. L. F., G. M. CAMPOS-TAKAKI \& J. L. LIMA FILHO, 1996. Effects of culture conditions on protease production by Streptomyces clavuligerus growing on soy bean flour medium. Applied Biochemistry and Biotechnology 60(2): 115-122. DOI: https://doi. org/10.1007/BF02788066.

RAO, M. B., A. M. TANKSALE, M. S. GHATGE \& V. V. DESHPANDE, 1998. Molecular and biotechnological aspects of microbial proteases. Microbiology and Molecular Biology Reviews 62(3): 597-635.

RAVEENDRAN, S., B. PARAMESWARAN, S. B. UMMALYMA, A. ABRAHAM, A. K. MATHEW, A. MADHAVAN \& A. PANDEY, 2018. Applications of microbial enzymes in food industry. Food Technology and Biotechnology 56(1): 16-30. DOI: http://dx.doi.org/10.17113/ $\mathrm{ftb} \cdot 56.01 .18 .5491$.

SAVITHA, S., S. SADHASIVAM, K. SWAMINATHAN \& F. H. LIN, 2011. Fungal protease: production, purification and compatibility with laundry detergents and their wash performance. Journal of the Taiwan Institute of Chemical Engineers 42(2): 298-304. DOI: https://doi.org/10.1016/j.jtice.2010.05.012.

SAYALI, K., P. SADICHHA \& S. SUREKHA, 2013. Microbial esterases: an overview. International Journal of Current Microbiology and Applied Sciences 2(7): 135-146.

SHELDON, R. A. \& S. VAN PELT, 2013. Enzyme immobilisation in biocatalysis: why, what and how. Chemical Society Reviews 42(15): 6223-6235. DOI: http://dx.doi.org/10.1039/C3CS60075K.

SINGH, R., A. MITTAL, M. KUMAR \& P. K. MEHTA, 2016. Microbial protease in commercial applications. Journal of Pharmaceutical, Chemical and Biological 4(3): 365-374.

SINGH, S. \& B. K. BAJAJ, 2017. Potential application spectrum of microbial proteases for clean and green industrial production. Energy, Ecology and Environment 2(6): 370-386. DOI: https:// doi.org/10.1007/s40974-017-0076-5.

ZHANG, X., Q. LIU, G. ZHANG, H. WANG \& T. NG, 2010. Purification and molecular cloning of a serine protease from the mushroom Hypsizigus marmoreus. Process Biochemistry 45(5): 724-730. DOI: https://doi.org/10.1016/j.procbio.2010.01.009.

ZIMMER, K. R., G. L. BORRÉ, D. S. TRENTIN, C. W. JÚNIOR, A. P. FRASSON, A. A. GRAEFF \& A. J. MACEDO, 2009. Enzimas microbianas de uso terapêutico e diagnóstico clínico. Revista Liberato 10(14): 123-137.

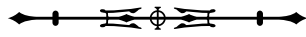


